\title{
Nissl Staining of Cat Spinal Cord is Significantly Influenced by Concentrations of Glutaraldehyde in Fixative
}

\author{
J. Chavez ${ }^{1}$, C. Graham ${ }^{1}$, A.S. Lossinsky ${ }^{1}$, R.L. Schultz ${ }^{2}$, and P.S. Manoonkitiwongsa ${ }^{1}$ \\ ${ }^{1}$ Neural Engineering Program, Huntington Medical Research Institutes, Pasadena, CA 91105 \\ ${ }^{2}$ Division of Human Anatomy, Loma Linda University, Loma Linda, CA 92507
}

The Nissl stain is a popular conventional light microscopic marker of neuronal cytoplasm $[1,2]$. The merit of the dye is in its ability to selectively stain Nissl bodies of neurons. Consequently, the stain enables visualization of neurons against a pale neuropil or white matter background. Alteration in Nissl body staining is commonly interpreted in pathology as indication of neuronal injury.

Some of our work involves light and electron microscopic evaluations of the same cat nervous tissue. Inclusion of glutaraldehyde with the phosphate-buffered formalin is needed for better preservation of the tissue for morphologic, ultrastructural studies. However, it is unclear whether Nissl staining would be affected by the presence of glutaraldehyde.

Thus, we compared Nissl staining of spinal cord tissue fixed by phosphate-buffered formalin alone or by formalin containing $0.25 \%$ or $3 \%$ glutaraldehyde. Following perfusion by the three fixatives, lumbar spinal cord tissues of adult cats were embedded in paraffin and $7 \mathrm{um}$ sections were stained by cresyl violet following standard Nissl protocol [3].

Formalin alone allowed a selective staining of neuronal cytoplasm. Although the fixative containing $0.25 \%$ glutaraldehyde yielded heavier staining of neurons, the neuropil was also stained. Formalin containing 3\% glutaraldehyde induced heavy staining of neurons, neuropil and the white matter.

These findings suggest that high concentrations of glutaraldehyde in the fixative should be avoided for Nissl staining of the spinal cord. Since pathologic evaluations of neuronal injury depend on proper Nissl staining, fixatives containing high concentrations of glutaraldehyde may complicate or bias interpretation of data. Fixation of spinal cord tissue by buffered formalin alone yields superior results. If glutaraldehyde is included in the fixative to allow for ultrastructural studies, antigen retrieval techniques prior to Nissl staining or modification of Nissl stain differentiation steps may be necessary to reduce the background.

\section{References}

[1] F.L. Carson, Histotechnology: A Self-Instructional Text, ASCP Press, Detroit, 1997.

[2] D.C. Sheenan, Theory and Practice of Histotechnology, C.V. Mosby Co., Missouri, 1980

[3] L.G. Luna, Manual of Histologic Staining Methods of the Armed Forces Institute of Pathology, McGraw-Hill, Inc., New York, 1968.

[4] This research was supported by NIH RO1-NS40860-02, NO1-DC-2105, NO1-NS-1-2340. The assistance of D.B. McCreery, V. Pikov, X. Liu, L. Bullara and W.F. Agnew is gratefully acknowledged. 

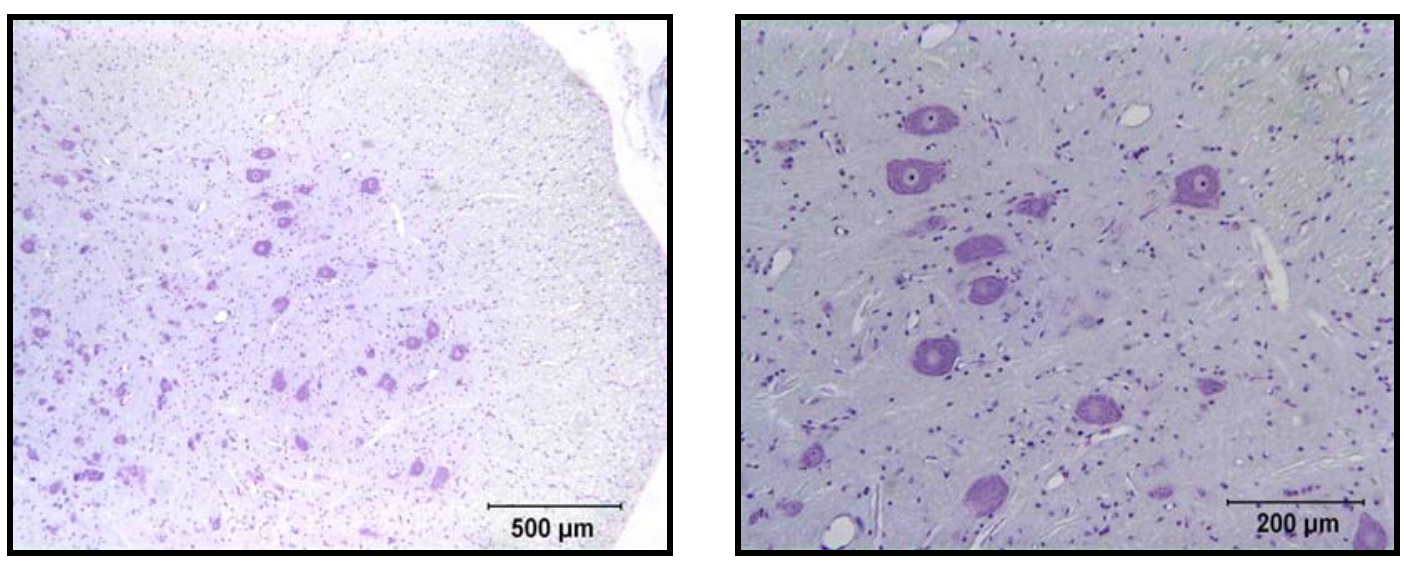

FIGURE 1. Low (left) and intermediate (right) magnifications of spinal cord fixed with formalin only.
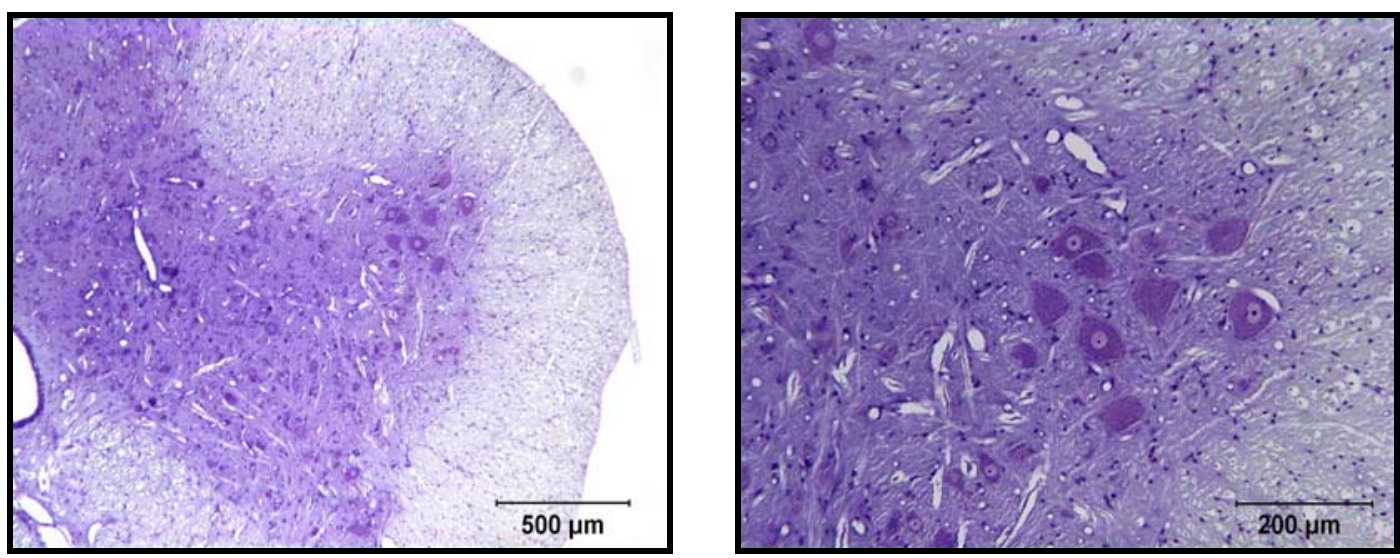

FIGURE 2. Low (left) and intermediate (right) magnifications of spinal cord fixed with formalin containing $0.25 \%$ glutaraldehyde.
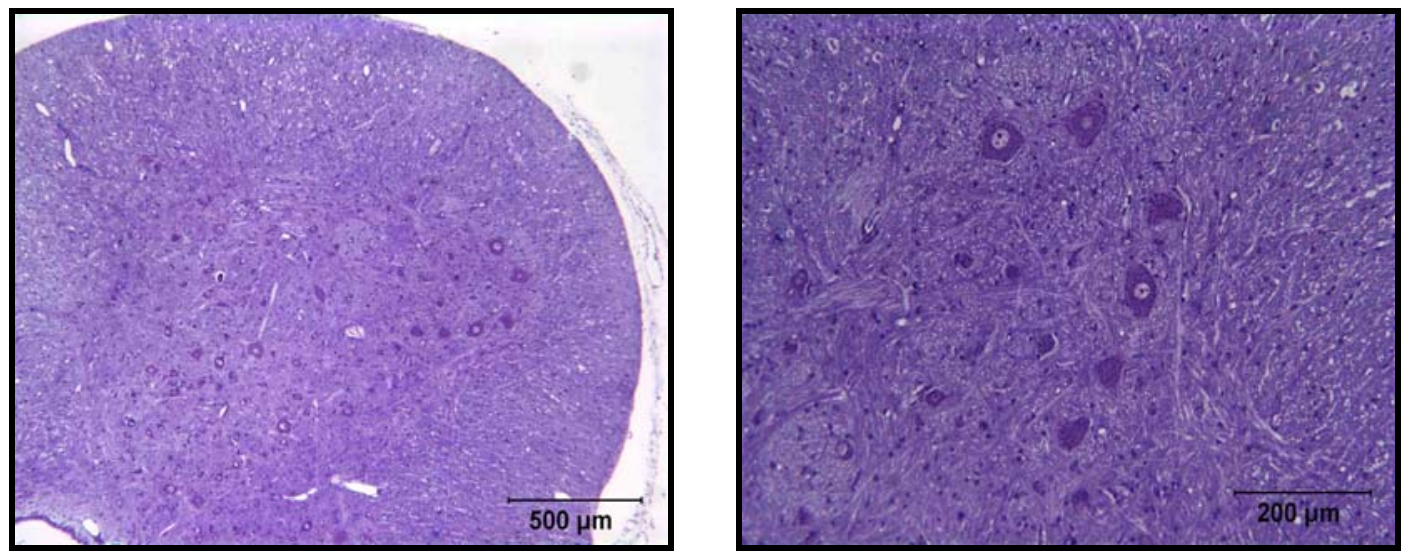

FIGURE 3. Low (left) and intermediate (right) magnifications of spinal cord fixed with formalin containing 3\% glutaraldehyde. 